\title{
Construction of Titanium-based Metal-Organic Frameworks based on the Ti/Cu Heteronuclear Cluster
}

\author{
Hao Fu, ${ }^{\mathrm{a}, \mathrm{b}, \mathrm{c}, \mathrm{d}}$ Qipu Lin, ${ }^{\mathrm{a}}$ Fei Wang, ${ }^{* \mathrm{a}}$ and Jian Zhang ${ }^{* a}$
}

aState Key Laboratory of Structural Chemistry, Fujian Institute of Research on the Structure of Matter, Chinese Academy of Sciences, 155 Yang Qiao West Road, Fuzhou, Fujian 350002, P. R. China

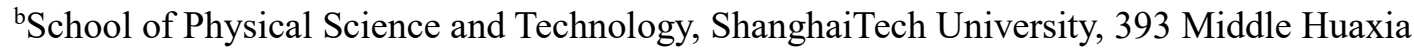
Road, Pudong, Shanghai 201210, China

'University of Chinese Academy of Sciences, 19 Yuquan Road, Shijingshan District, Beijing 100049, China

${ }^{\text {d} S h a n g h a i ~ A d v a n c e d ~ R e s e a r c h ~ I n s t i t u t e, ~ C h i n e s e ~ A c a d e m y ~ o f ~ S c i e n c e s, ~} 99$ Haike Road, Zhangjiang Hi-Tech Park, Pudong, Shanghai 201210, China

*Corresponding Author: Email: wangfei04@fjirsm.ac.cn and zhj@fjirsm.ac.cn 


\section{Materials and Instrumentation}

All chemical reagents were obtained from commercial supplies without further purification. Fourier transform infrared spectroscopy (FT-IR) data were collected on a PerkinElmer Spectrum 100 FT-IR Spectrometer. UV-Vis absorption spectra were measured on a Perkin-Elmer Lambda 950 UV-Vis spectrophotometer. Powder X-ray diffraction (XRD) data were collected on a Rigaku Mini Flex II diffractometer using $\mathrm{Cu} \mathrm{K} \alpha$ radiation $(\lambda=1.54056 \AA)$ under ambient conditions. The X-ray photoelectron spectroscopy (XPS) analysis were collected on a ESCALAB 250Xi. The thermogravimetric analyses (TGA) were performed on a Mettler Toledo TGA/SDTA 851e analyzer in air atmosphere with a heating rate of 10 ${ }^{\circ} \mathrm{C} / \min$ from $30{ }^{\circ} \mathrm{C}$ to $800{ }^{\circ} \mathrm{C}$.

Caution! Pyridine has strong volatility and toxicity, so precautions with suitable care and protection for synthesis of compounds 1 and 2 have been followed. Besides, it is necessary to pay attention to the volume of solvent in glass bottles or polytetrafluoroethylene reactor when conducting high temperature solvothermal to avoid explosion.

\section{Synthesis of 1 .}

$\mathrm{CuSO}_{4}(0.200 \mathrm{~g}, 1.25 \mathrm{mmol})$, benzoic acid $(0.300 \mathrm{~g}, 2.46 \mathrm{mmol})$, pyridine $(0.200 \mathrm{~mL}, 2.48 \mathrm{mmol}) \mathrm{N}, \mathrm{N}-$ dimethylformamide $(5.0 \mathrm{~mL})$ were mixed at room temperature; then Titanium(IV) isopropoxide $\left(\mathrm{Ti}(\mathrm{O} \text { i } \mathrm{Pr})_{4}\right)(0.5 \mathrm{ml}$, $1.6 \mathrm{mmol}$ ) was added. The resultant solution was heated at $120^{\circ} \mathrm{C}$ for two days. After cooled to room temperature, green crystals of 1 were obtained. Yield: $\sim 120 \mathrm{mg}\left(\sim 34 \%\right.$ based on $\left.\mathrm{CuSO}_{4}\right)$. FTIR $\left(\mathrm{KBr}, \mathrm{cm}^{-1}\right)$ : $v=3477(\mathrm{~m}), 3119$ (w),3 077 (w), 3049 (w), 2860 (w), 1655 (s), 1605 (m), 1509 (m), 1490 (m), 1449 (m), 1404 (s), 1246(s), 1060 (s), $1036(\mathrm{~s}), 957(\mathrm{~s})$

\section{Synthesis of 2.}

$\mathrm{CuSO}_{4}(0.200 \mathrm{~g}, 1.25 \mathrm{mmol})$, phenol $(0.300 \mathrm{ml}, 2.46 \mathrm{mmol})$, pyridine $(0.200 \mathrm{~mL}, 2.48 \mathrm{mmol})$, N,Ndimethylformamide $(5.0 \mathrm{~mL})$ were mixed at room temperature; then $\mathrm{Ti}(\mathrm{O} \mathrm{Pr})_{4}(0.5 \mathrm{~mL}, 1.6 \mathrm{mmol})$ was added. The resultant solution was heated at $120^{\circ} \mathrm{C}$ for one day. After cooled to room temperature, green crystals of 2 were obtained. Yield: $\sim 150 \mathrm{mg}\left(\sim 38 \%\right.$ based on $\left.\mathrm{CuSO}_{4}\right)$. FTIR $\left(\mathrm{KBr}, \mathrm{cm}^{-1}\right): v=3481(\mathrm{~m}), 3219(\mathrm{w}), 3116(\mathrm{w}), 3099(\mathrm{w})$, $2930(\mathrm{w}), 1651$ (s), 1601 (m), 1488 (m), 1445 (m), 1228 (s), 1204 (s), 1083 (s), 1064 (s), 961 (s).

\section{Synthesis of 3 .}

$\mathrm{CuSO}_{4}(0.200 \mathrm{~g}, 1.25 \mathrm{mmol})$, 4-dimethylaminopyridine $(0.250 \mathrm{~g}, 2.05 \mathrm{mmol})$, N,N-dimethylformamide $(3.0 \mathrm{~mL})$ and triethylene glycol $(2.0 \mathrm{~mL})$ were mixed at room temperature; then $\mathrm{Ti}\left(\mathrm{O}^{\mathrm{i} P r}\right)_{4}(0.5 \mathrm{~mL}, 1.6 \mathrm{mmol})$ was added. The resultant solution was heated at $120^{\circ} \mathrm{C}$ for two days. After cooled to room temperature, green crystals of $\mathbf{3}$ were obtained. Yield: $\sim 100 \mathrm{mg}\left(\sim 24 \%\right.$ based on $\left.\mathrm{CuSO}_{4}\right)$. FTIR $\left(\mathrm{KBr}, \mathrm{cm}^{-1}\right): \mathrm{v}=3486(\mathrm{w}), 3219(\mathrm{w}), 3084(\mathrm{w}), 3060(\mathrm{w})$, 2928 (w), 1641 (s), 1614 (s), 1538 (m), 1450 (m), 1390 (m), 1228 (s), 1197 (s), 1066 (s), 1021 (s), 948 (s). 


\section{Crystallographic studies}

The X-ray single crystal diffraction data of $\mathbf{1}$ and $\mathbf{2}$ was collected on a Synergy Custom (Liquid MetalJet $\mathrm{D} 2+)$ with graphite-monochromatic $\mathrm{Ga} \mathrm{K} \alpha$ radiation $(\lambda=1.3405 \AA)$. The X-ray single crystal diffraction data of 3 were collected on a MM007 with graphite-monochromaticc Mo Ka radiation $(\lambda=0.7103 \AA)$. The Structure solutions and refinements were done with the OLEX2. ${ }^{1}$ Contributions to scattering due to disordered solvent molecules were removed using the SQUEEZE routine of PLATON. ${ }^{2}$

Table S1 Crystallographic data of 1 to 3

\begin{tabular}{|c|c|c|c|}
\hline Compound Reference & 1 & 2 & 3 \\
\hline Chemical Formula & $\mathrm{C}_{54} \mathrm{H}_{70} \mathrm{Cu}_{6} \mathrm{~N}_{10} \mathrm{O}_{49} \mathrm{~S}_{8} \mathrm{Ti}_{6}$ & $\mathrm{C}_{65} \mathrm{H}_{75} \mathrm{Cu}_{10} \mathrm{~N}_{14} \mathrm{O}_{53} \mathrm{~S}_{10} \mathrm{Ti}_{6}$ & $\mathrm{C}_{96} \mathrm{H}_{141} \mathrm{Cu}_{9} \mathrm{~N}_{27} \mathrm{O}_{55} \mathrm{~S}_{11} \mathrm{Ti}_{6}$ \\
\hline Formula Mass & 2568.32 & 3143.79 & 3693.25 \\
\hline Crystal System & orthorhombic & monoclinic & trigonal \\
\hline $\mathrm{a} / \AA$ & $51.9916(10)$ & $32.3551(10)$ & $26.4068(7)$ \\
\hline $\mathrm{b} / \AA$ & $25.9771(5)$ & $14.4492(4)$ & $26.4068(7)$ \\
\hline $\mathrm{c} / \AA$ & $14.4751(2)$ & $27.4831(8)$ & $19.2680(7)$ \\
\hline$\alpha /^{\circ}$ & 90 & 90 & 90 \\
\hline$\beta /{ }^{\circ}$ & 90 & $110.223(4)$ & 90 \\
\hline$\gamma /{ }^{\circ}$ & 90 & 90 & 120 \\
\hline Volume/ $\AA^{3}$ & $19549.9(6))$ & $12056.4(7)$ & $11635.9(7)$ \\
\hline$\rho_{\text {calc }} \mathrm{g} / \mathrm{cm}^{3}$ & 1.745 & 1.732 & 1.581 \\
\hline$\mu / \mathrm{mm}^{-1}$ & 10.988 & 12.896 & 1.728 \\
\hline Temperature/K & $106(6)$ & $100.3(8)$ & $293(2)$ \\
\hline Space Group & Fdd 2 & $\mathrm{C} 2 / \mathrm{c}$ & $\mathrm{R} 3$ \\
\hline $\mathrm{Z}$ & 8 & 4 & 3 \\
\hline $\begin{array}{c}\text { No. of Reflections } \\
\text { Measured }\end{array}$ & 12359 & 30869 & 35001 \\
\hline $\begin{array}{l}\text { No. of Independent } \\
\text { Reflections }\end{array}$ & 6045 & 9567 & 17797 \\
\hline Rint & 0.0758 & 0.0652 & 0.0871 \\
\hline $\begin{array}{l}\text { Final R1 Values } \\
\qquad(\mathrm{I}>2 \sigma(\mathrm{I}))\end{array}$ & 0.0981 & 0.0892 & 0.0676 \\
\hline $\begin{array}{l}\text { Final wR(F2) Values } \\
\qquad(\mathrm{I}>2 \sigma(\mathrm{I}))\end{array}$ & 0.2766 & 0.2648 & 0.1523 \\
\hline Goodness of Fit on F2 & 1.152 & 1.067 & 0.948 \\
\hline Flack parameter & $0.026(15)$ & l & $0.053(18)$ \\
\hline CCDC Number & 2041715 & 2041716 & 2041717 \\
\hline
\end{tabular}




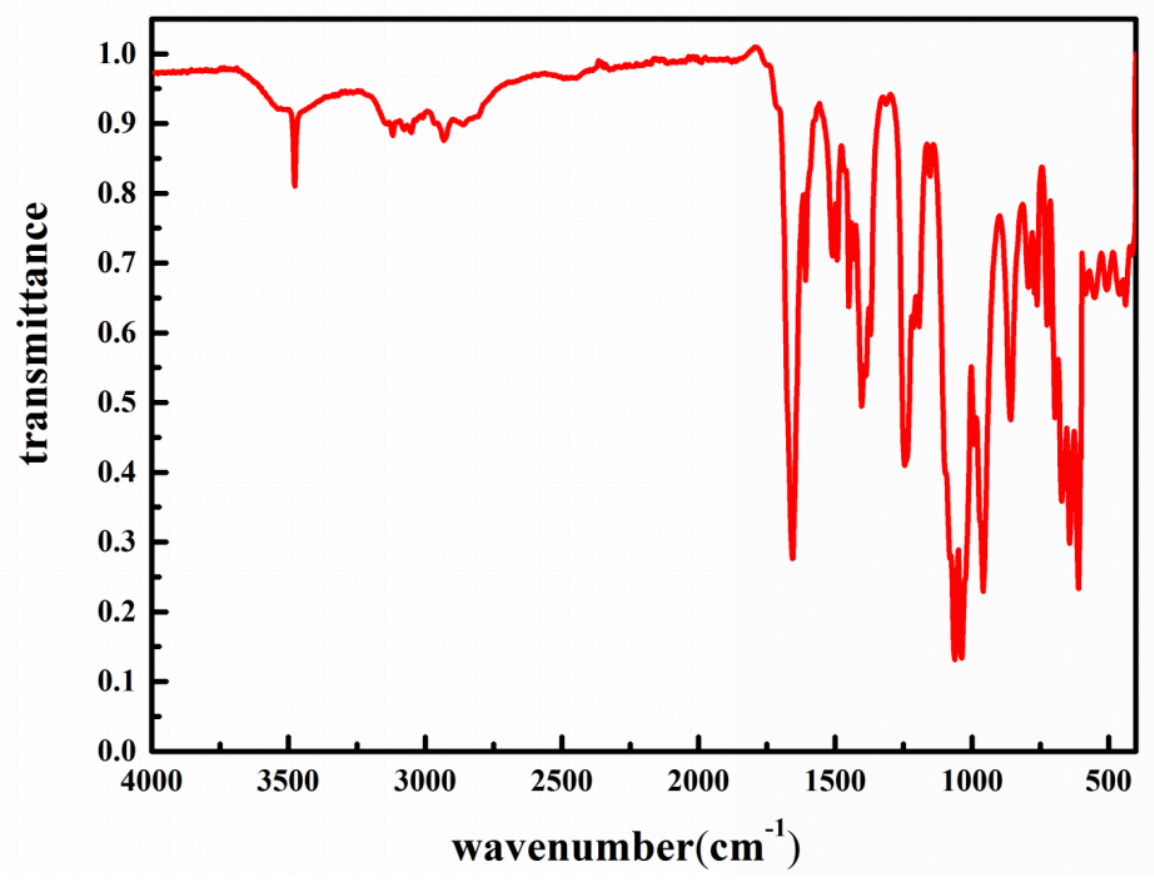

Fig. S1. FT-IR spectra of 1 .

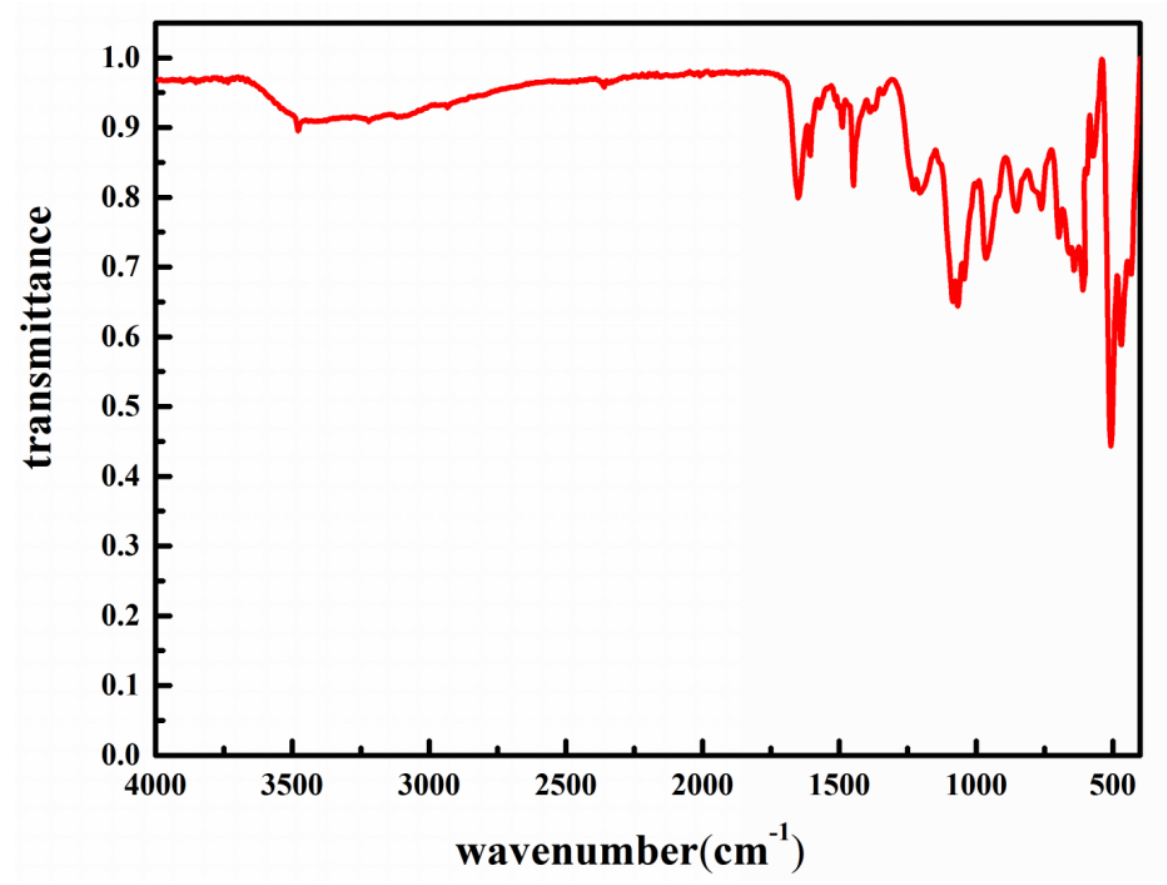

Fig. S2. FT-IR spectra of 2. 


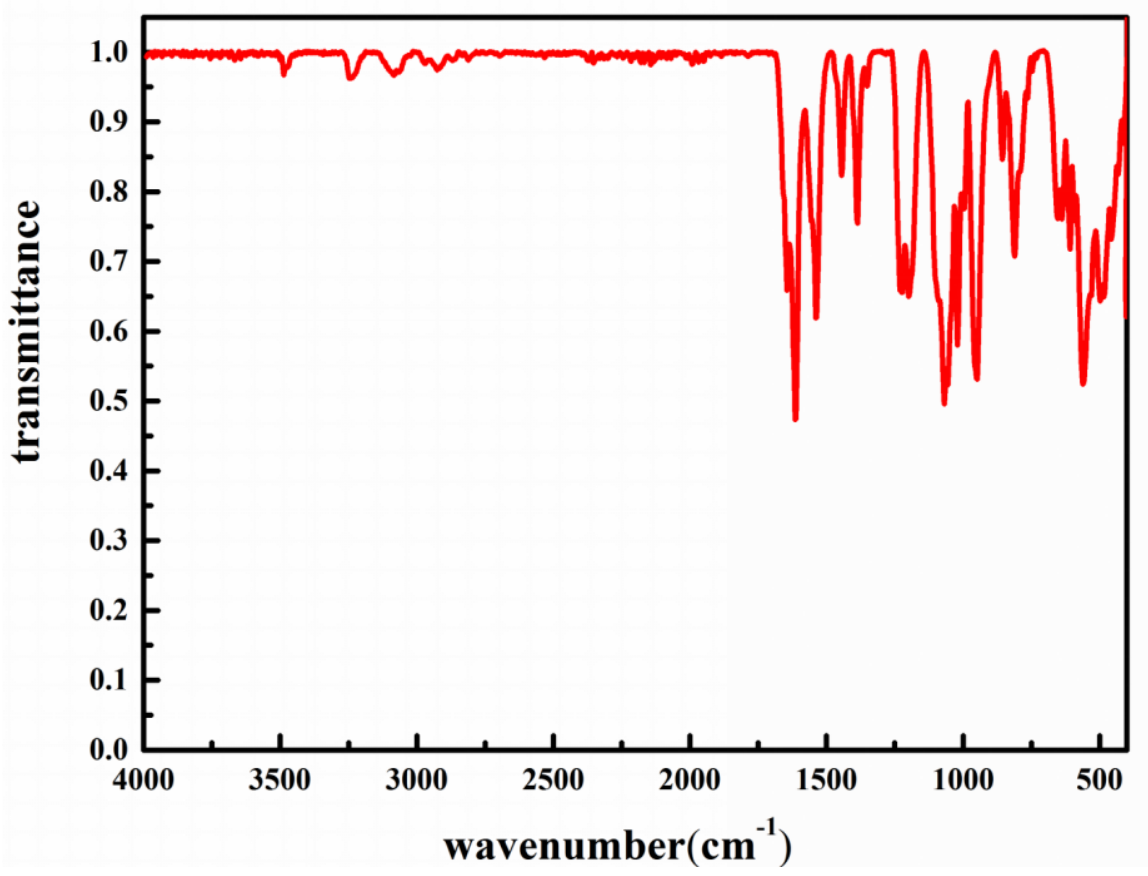

Fig. S3. FT-IR spectra of 3.

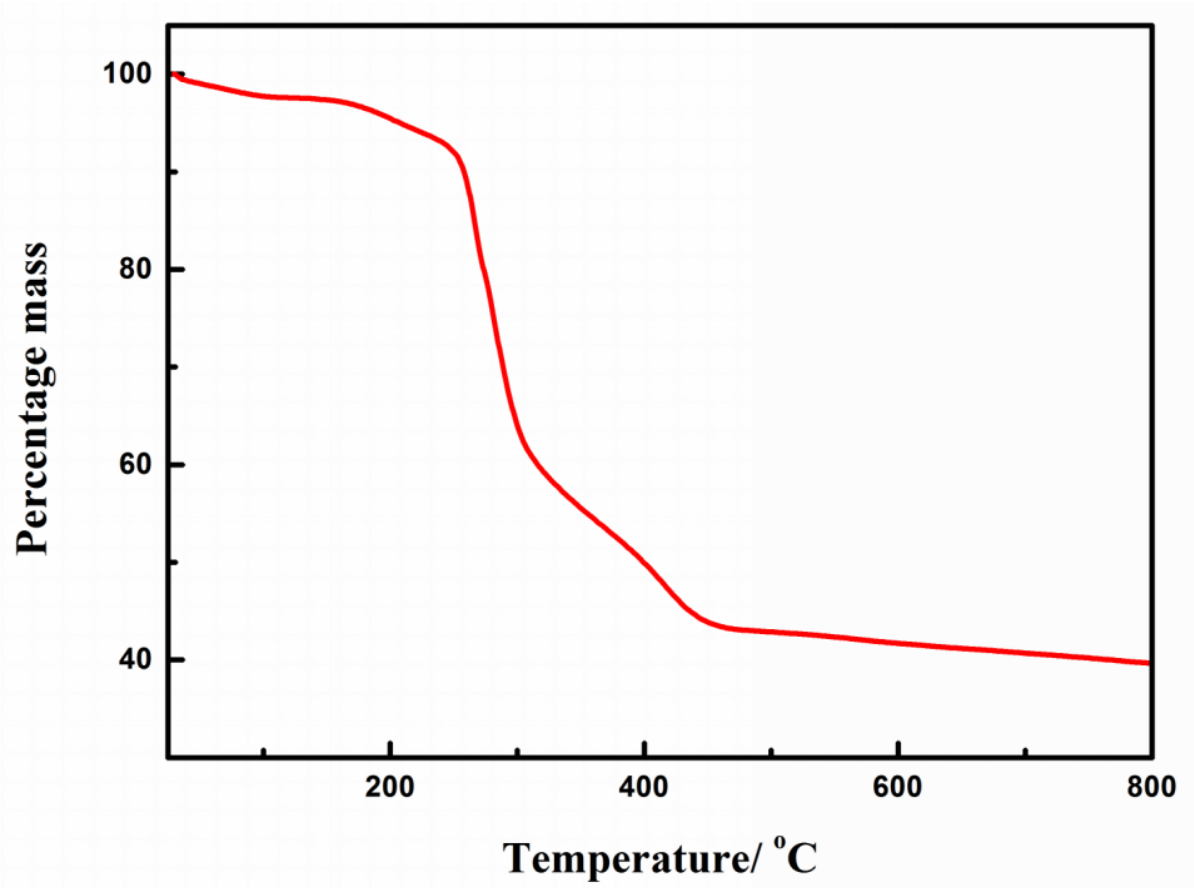

Fig. S4. TGA curves of 1. 


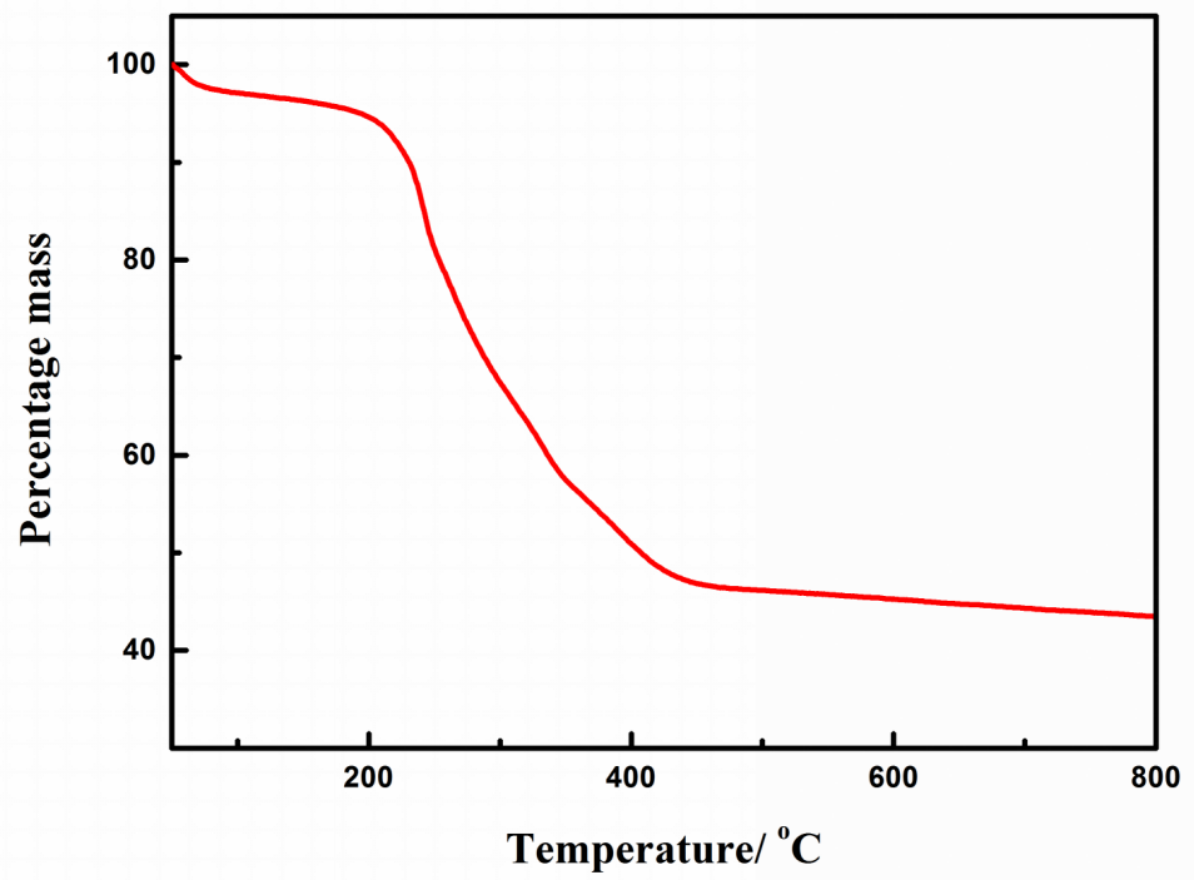

Fig. S5. TGA curves of 2.

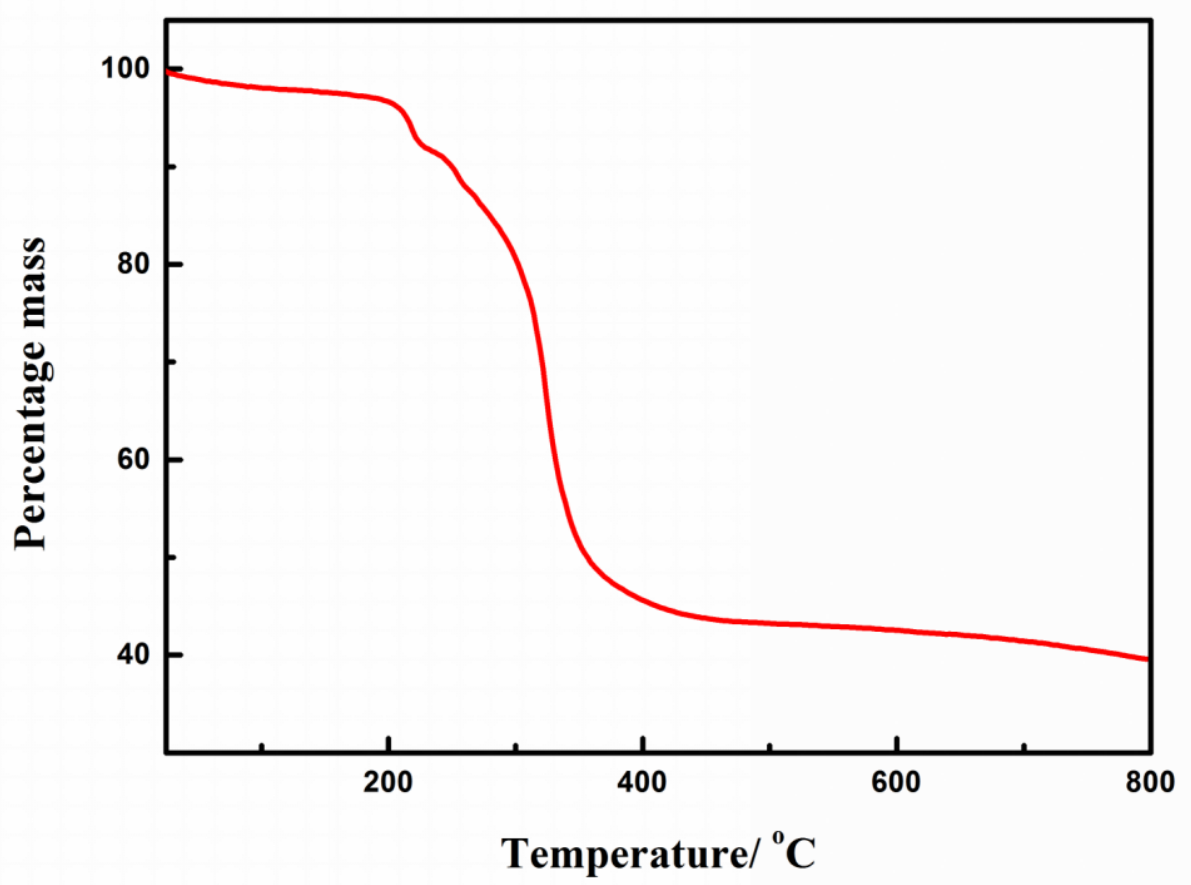

Fig. S6. TGA curves of 3. 


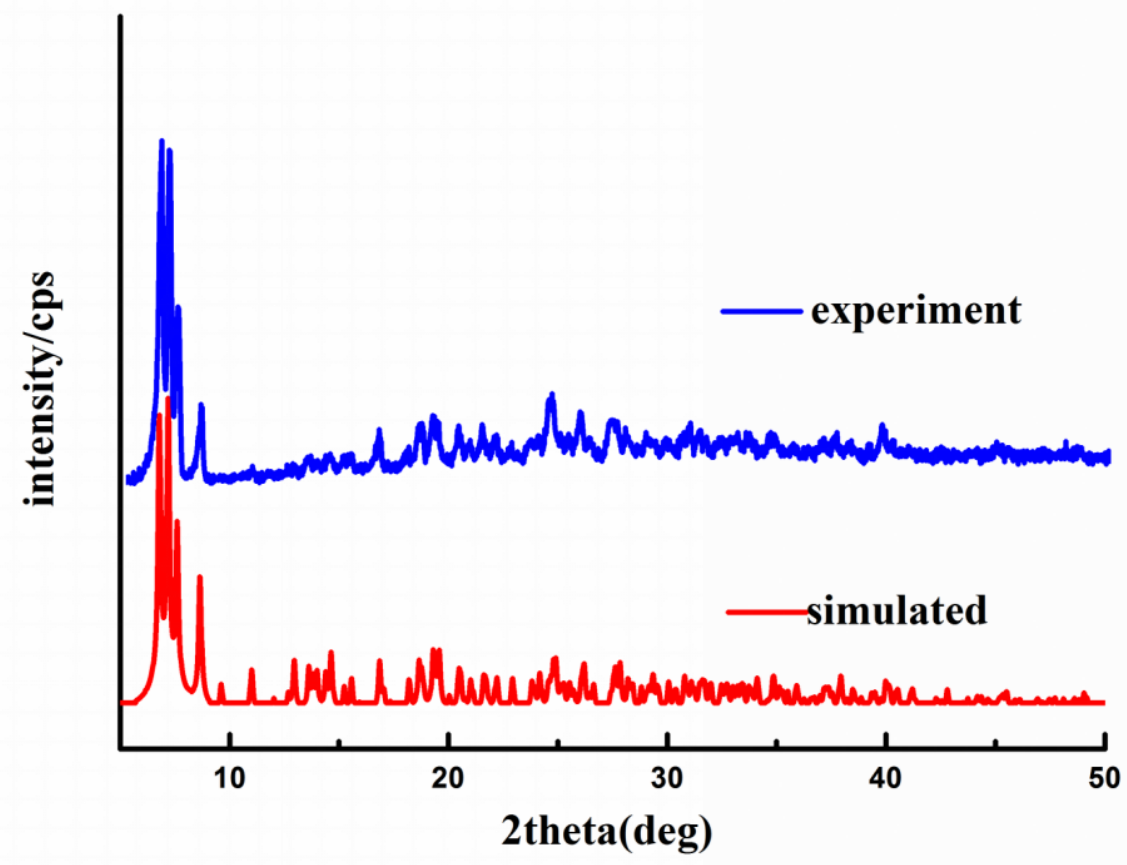

Fig. S7. PXRD patterns of 1.

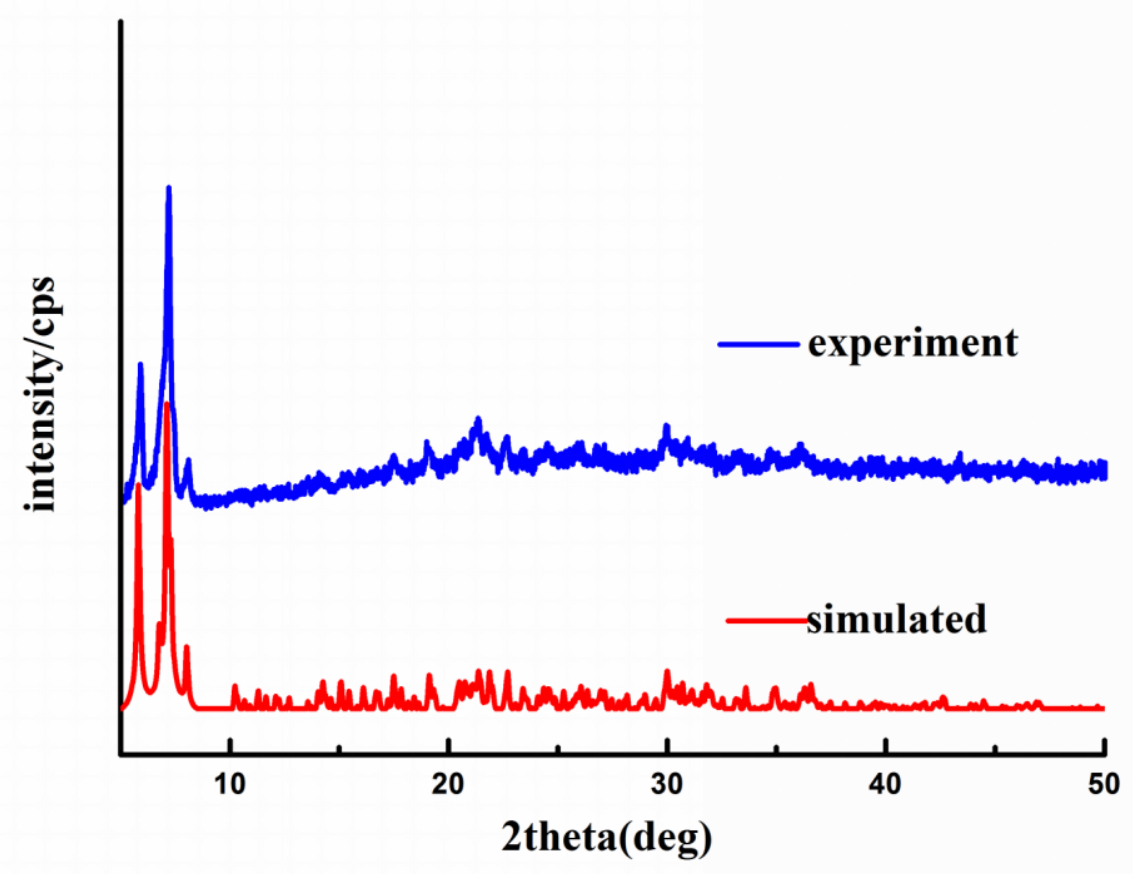

Fig. S8. PXRD patterns of 2. 


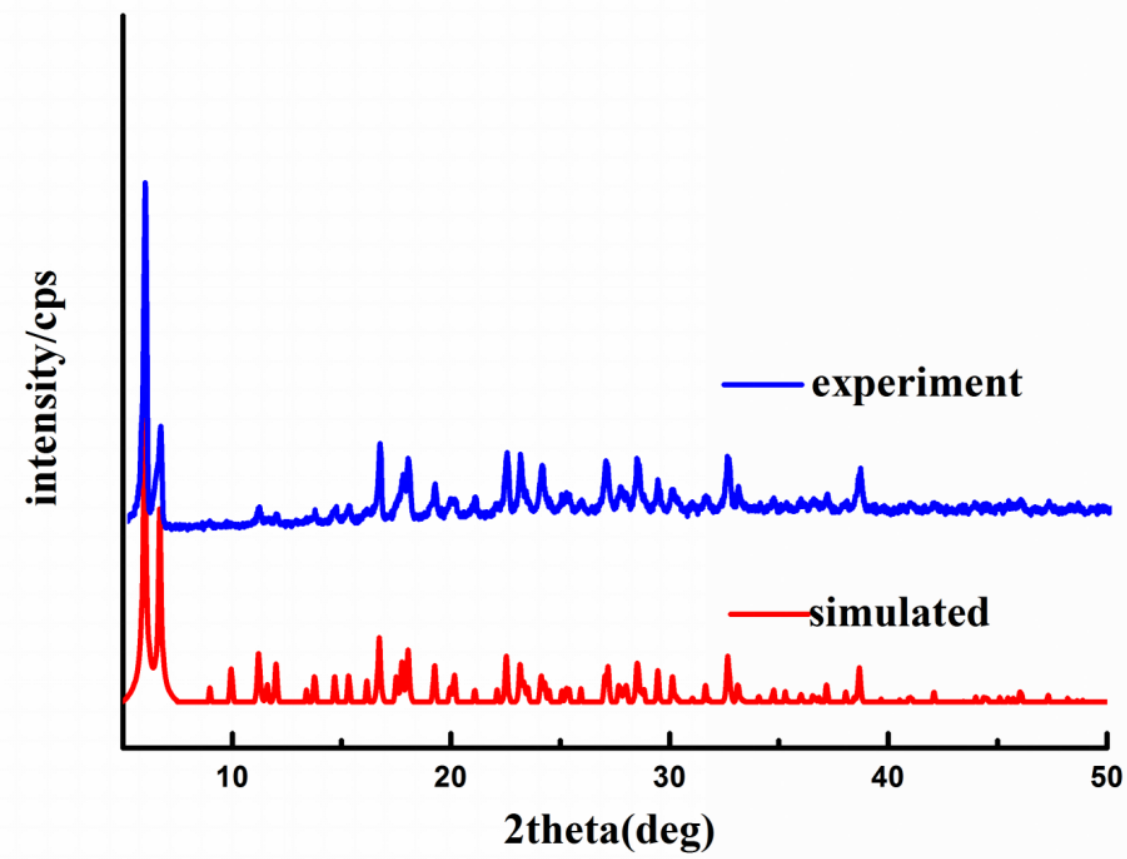

Fig. S9. PXRD patterns of 3.

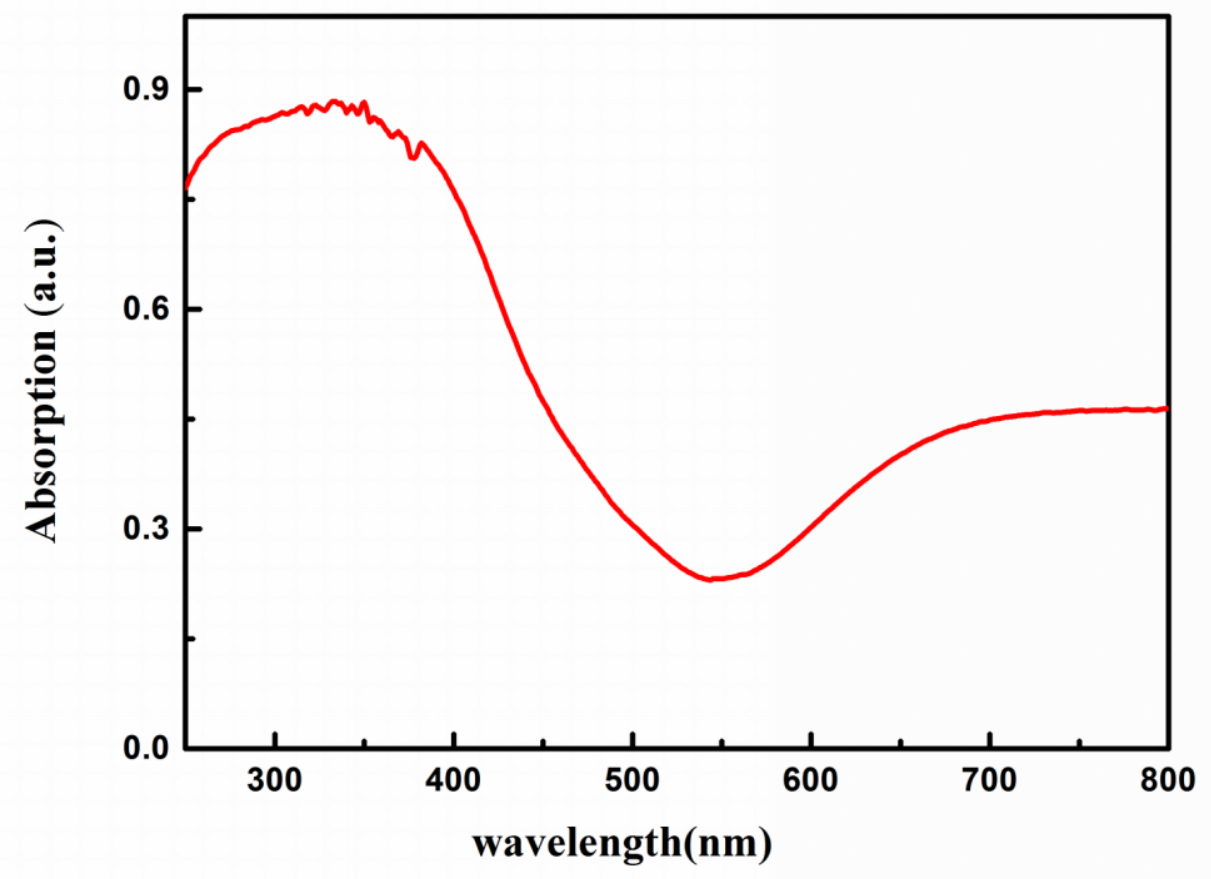

Fig. S10. UV-vis patterns of 1. 


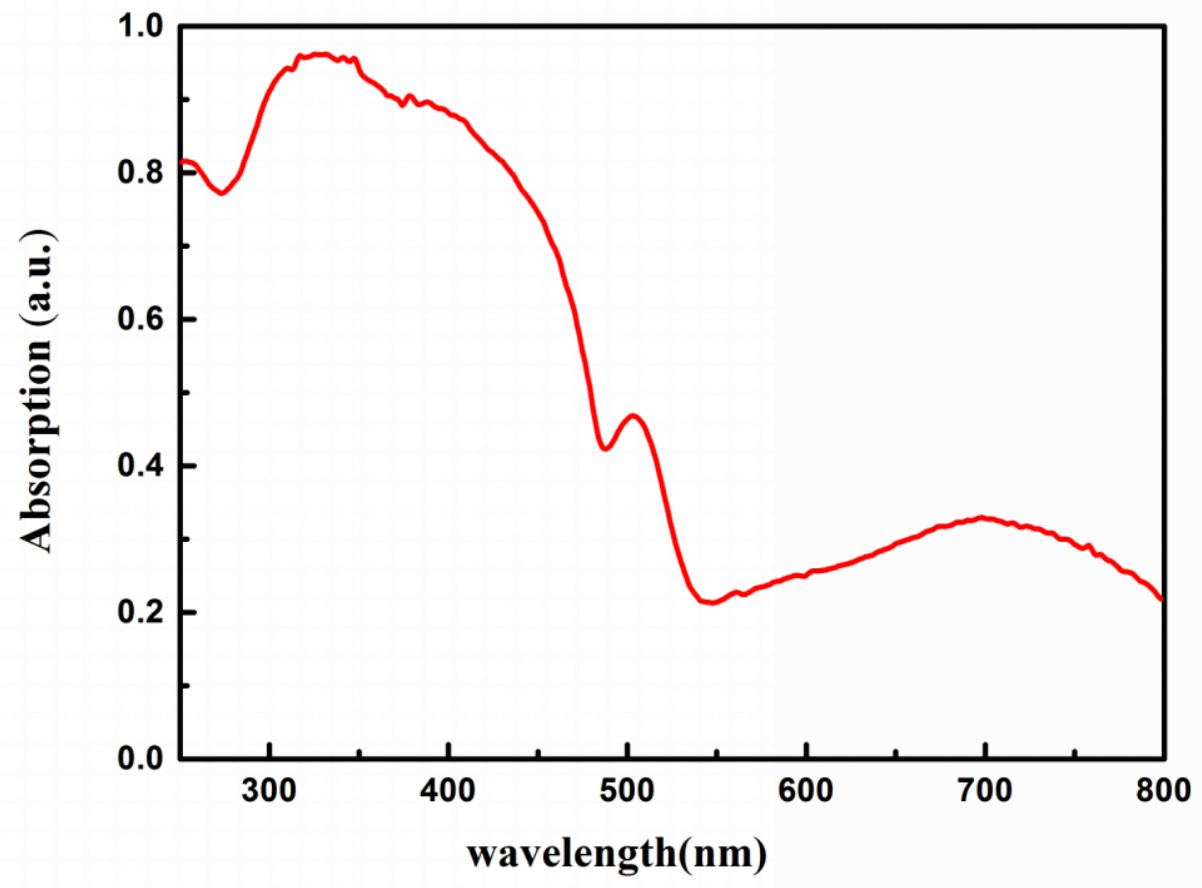

Fig. S11. UV-vis patterns of 2.

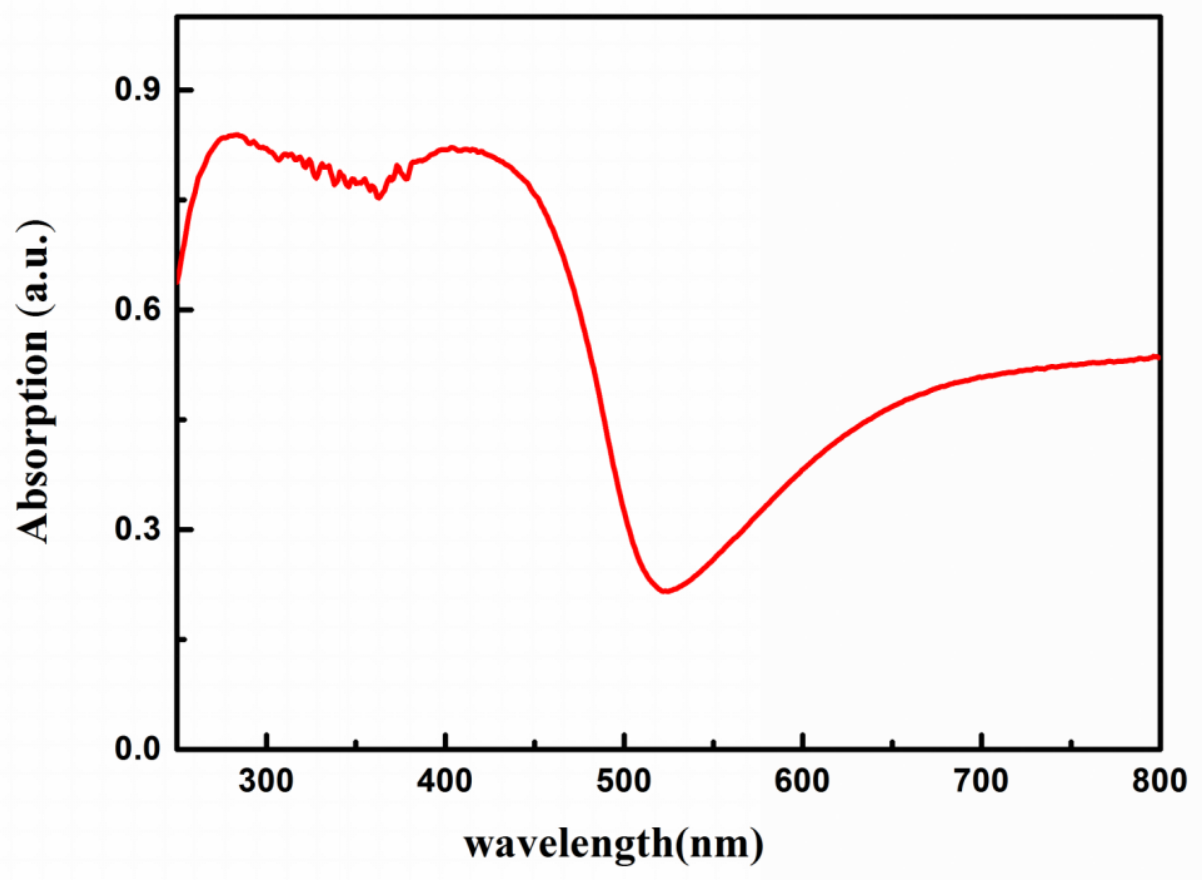

Fig. S12. UV-vis patterns of 3. 


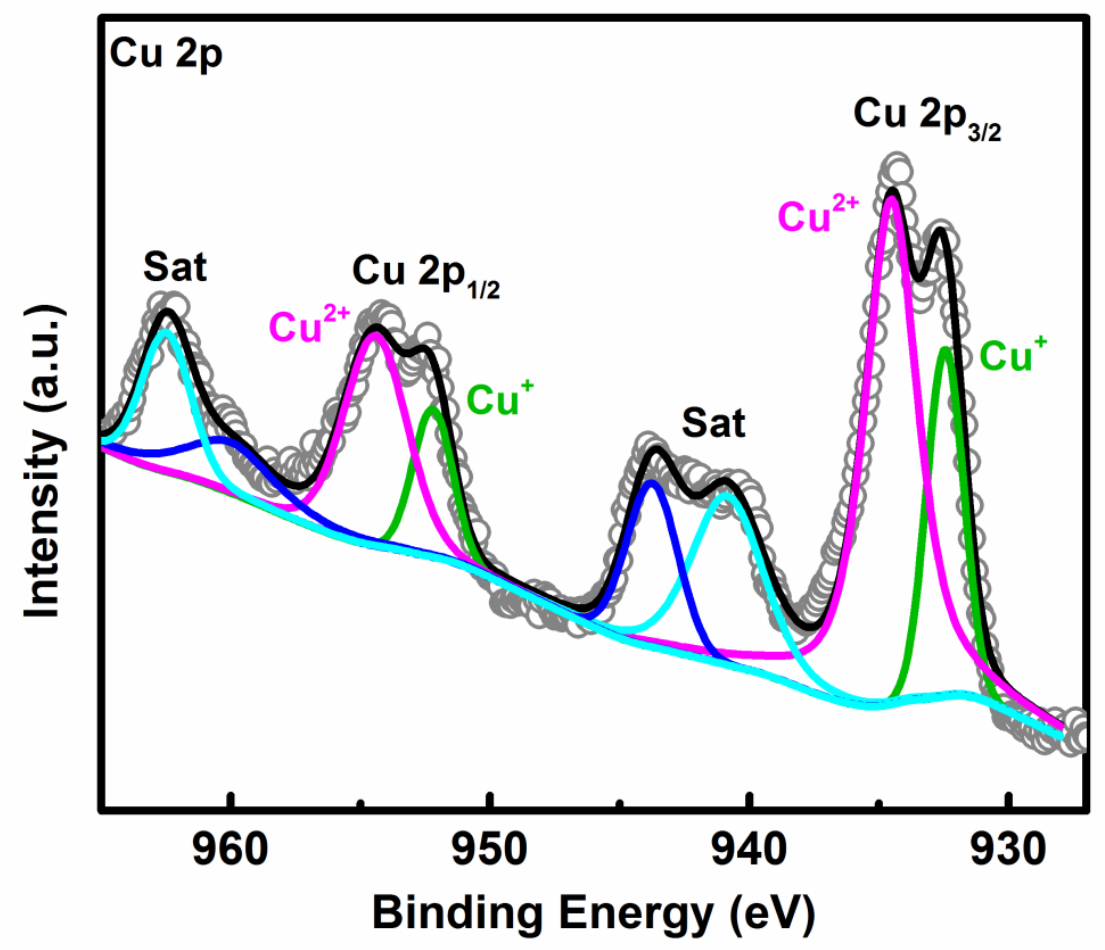

Fig. S13. XPS of $\mathrm{Cu}$ of 2.

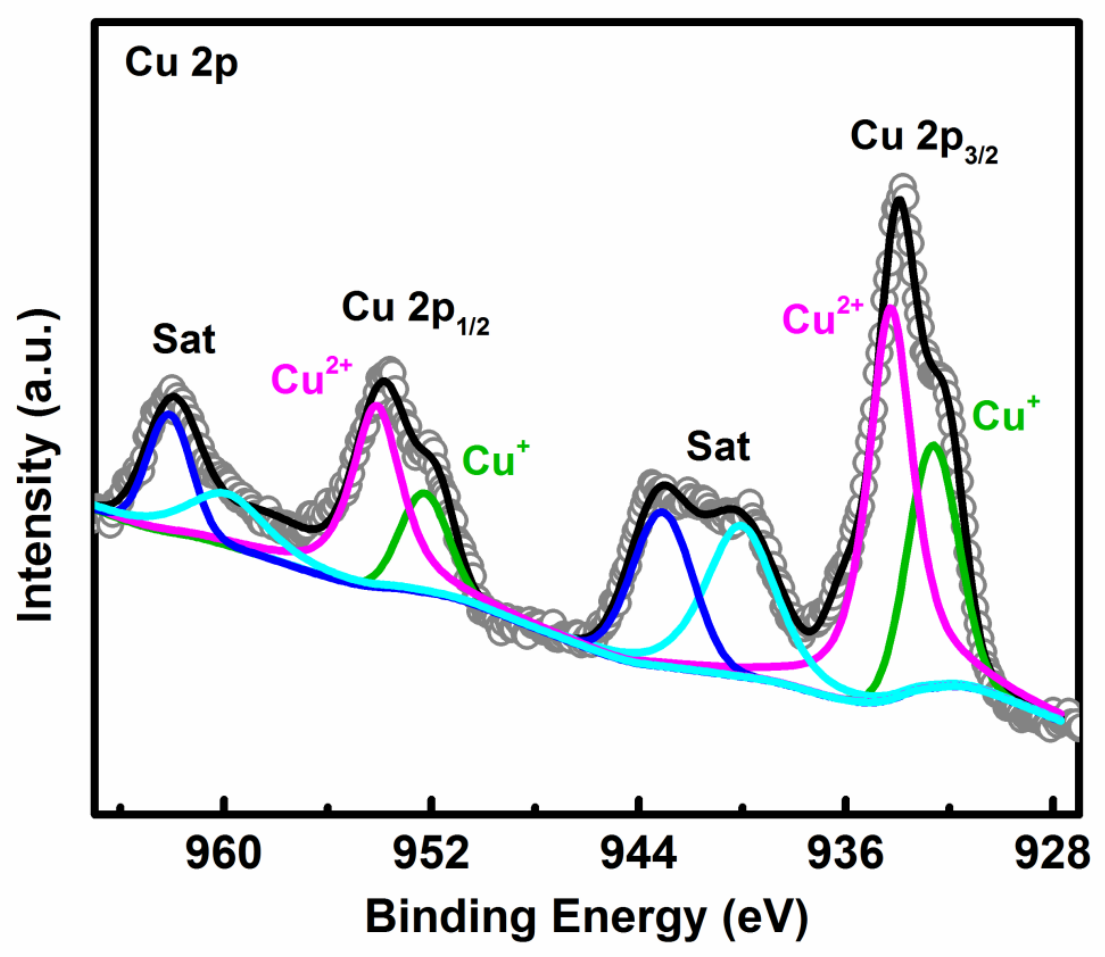

Fig. S14. XPS of $\mathrm{Cu}$ of 3. 


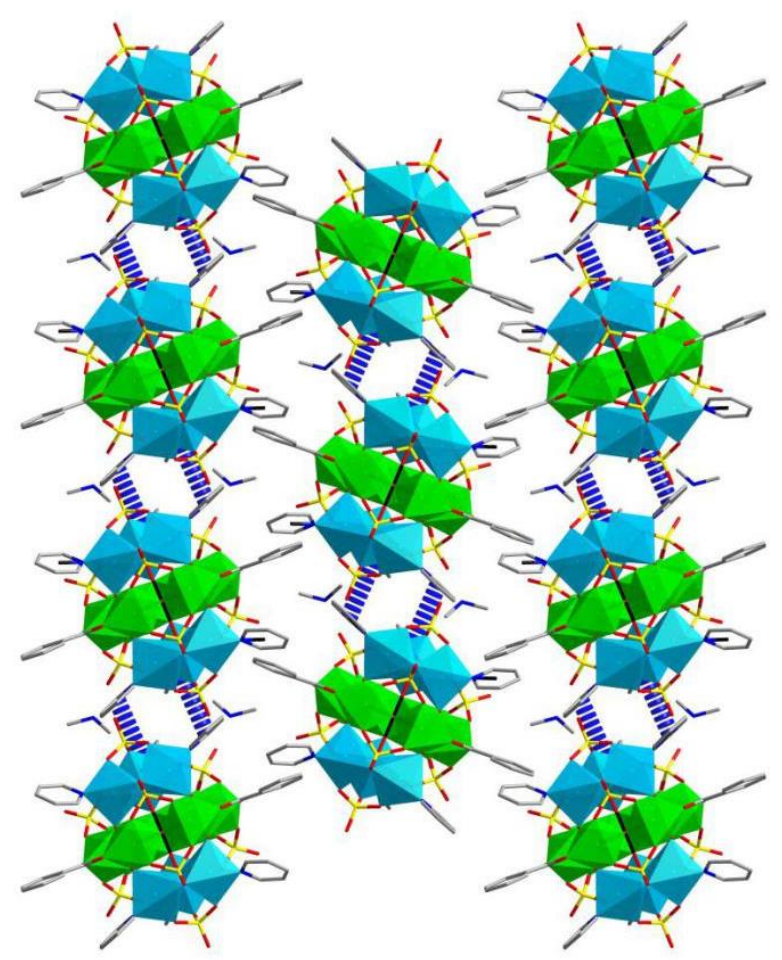

Fig. S15. The pack mode and intermolecular hydrogen bonding of $\mathbf{1}$.

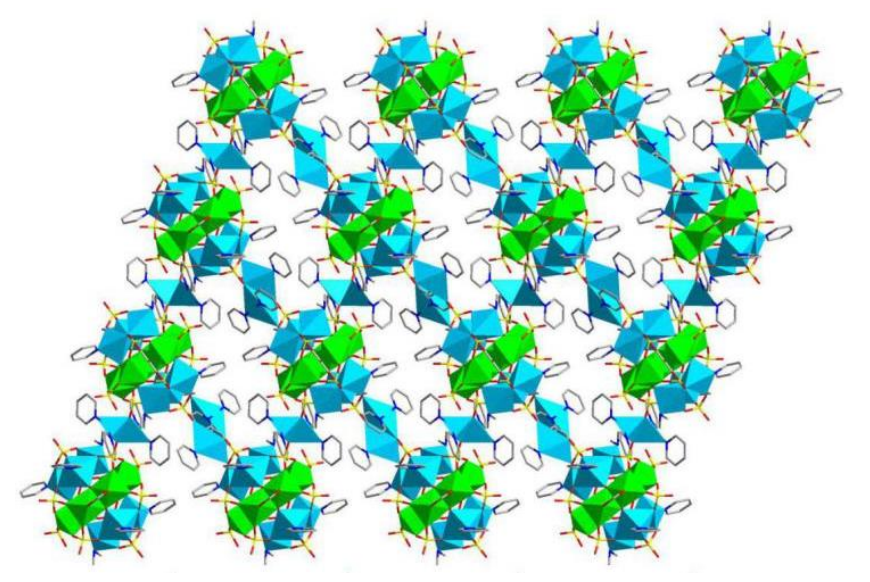

Fig. S16. The structure of framework of 2.

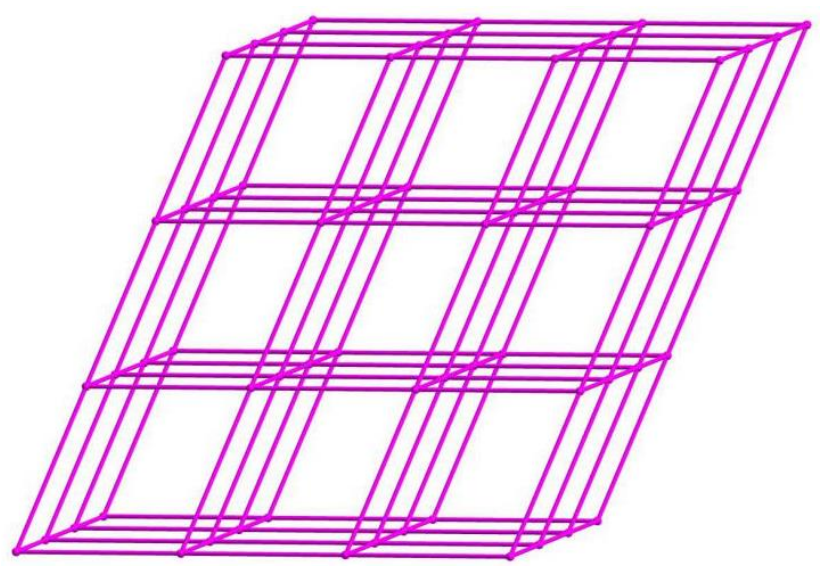

Fig. S17. The pcu topology structure of $\mathbf{3}$. 
(a)

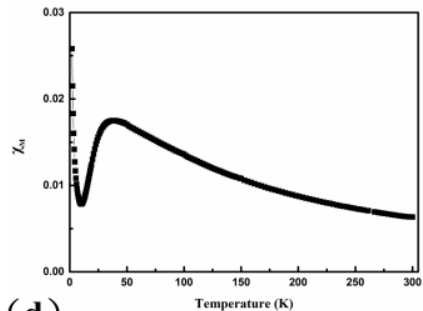

(d)

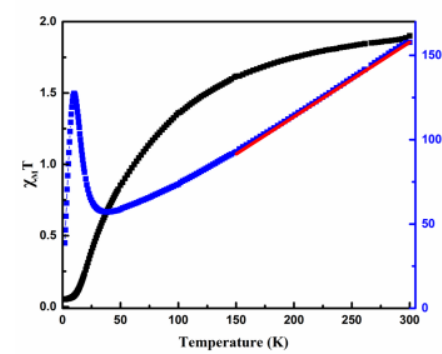

(b)
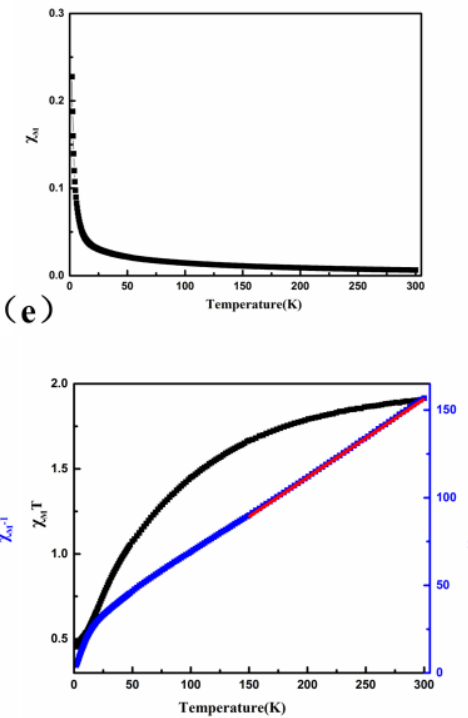

(c)
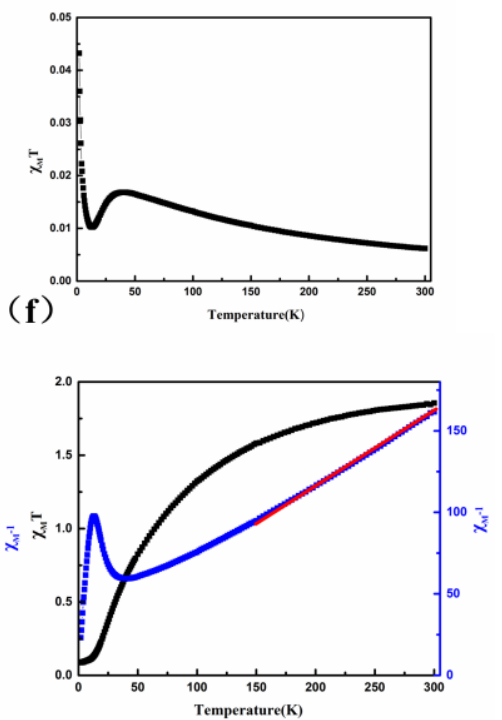

Fig. S18. Temperature dependencies of $\chi \mathrm{M}, \chi \mathrm{M}-1$ and $\chi \mathrm{MT}$ (inset) at $1 \mathrm{kOe}$ for $\mathbf{1}$ (a) (d) /2 (b) (e) /3 (c) (f). The red solid lines are the fit to the Curie-Weiss law.

Table S2 Bond valence sum (BVS) analysis of metal ions in $\mathbf{1}^{[\mathrm{a}]}$

Cu1 2.113

Cu1-O3 $0.097 \quad \mathrm{~d}=2.543(17)$

Cu1-O9 $0.483 \quad \mathrm{~d}=1.948(16)$

$\mathrm{Cu} 1-\mathrm{O} 12 \quad 0.474 \quad \mathrm{~d}=1.955(17)$

$\mathrm{Cu} 1-\mathrm{O} 14 \quad 0.157 \quad \mathrm{~d}=2.364(18)$

$\mathrm{Cu} 1-\mathrm{O} 20^{1} \quad 0.375 \mathrm{~d}=2.042(15)$

$\mathrm{r}_{1}=1.679$

Cu1-N1 $0.527 \quad \mathrm{~d}=1.992(17)$

$\mathrm{r}_{1}=1.751$

\section{$\mathrm{Cu} 22.005$}

$\mathrm{Cu} 2-\mathrm{O} 11^{1} \quad 0.407 \quad \mathrm{~d}=2.012(18)$

$\mathrm{Cu} 2-\mathrm{O} 14^{1} \quad 0.103 \quad \mathrm{~d}=2.521(16)$

Cu2-O19 $0.426 \quad \mathrm{~d}=1.995(16)$

$\mathrm{Cu} 2-\mathrm{O} 20 \quad 0.412 \quad \mathrm{~d}=2.007(16)$

$\mathrm{Cu} 2-\mathrm{O} 22 \quad 0.122 \quad \mathrm{~d}=2.458(17)$

$r_{1}=1.679$

Cu2-N3 $0.536 \quad \mathrm{~d}=1.982(11)$

$\mathrm{r}_{1}=1.751$

\section{Cu3 2.104}

Cu3-O21 $0.128 \quad \mathrm{~d}=2.439(17)$

Cu3-O8 $0.473 \quad d=1.956(16)$

${\mathrm{Cu} 3-\mathrm{O} 10^{1}}^{1} 0.430 \quad \mathrm{~d}=1.991(17)$

$\mathrm{Cu} 3-\mathrm{O} 20 \quad 0.417 \quad \mathrm{~d}=2.003(16)$

$\mathrm{Cu} 3-\mathrm{O} 22 \quad 0.118 \quad \mathrm{~d}=2.471(18)$

$\mathrm{r}_{1}=1.679$ 
Cu3-N2 $0.539 \quad d=1.980(2)$

$\mathrm{r}_{1}=1.751$

${ }^{\text {[a] }} \mathrm{Vi}=\Sigma \mathrm{Sij}=\Sigma \exp [(\mathrm{r} 1-\mathrm{rij}) / \mathrm{B}], \mathrm{r} 1$ is the bond length between atoms $\mathrm{i}$ and $\mathrm{j}$ (here $\mathrm{r} 1=1.679$ for $\mathrm{Cu} \mathrm{u}^{\mathrm{ii}} \mathrm{O}$; $\mathrm{r} 1=1.751$ for $\left.\mathrm{Cu}^{\mathrm{ii}}-\mathrm{N}\right)$; B is a constant, the "universal parameter" $\sim 0.37 \AA$; $\mathrm{Sij}$ is the valence of a bond between atoms $\mathrm{i}$ and $\mathrm{j}$; Vi is the sum of all bond valences of the bonds formed by a given atom $\mathrm{i}^{3}$

Table S3 Bond valence sum (BVS) analysis of metal ions in $2 .{ }^{[b]}$

Cu1 2.090

Cu1-O28 $0.397 \quad \mathrm{~d}=2.020(5)$

Cu1-O4 $0.464 \quad \mathrm{~d}=1.963(6)$

Cu1-O6 $0.114 \quad d=2.484(6)$

Cu1-O15 $0.464 \quad \mathrm{~d}=1.963(5)$

$\mathrm{Cu} 1-\mathrm{O} 22^{1} \quad 0.102 \quad \mathrm{~d}=2.524(6)$

$\mathrm{r}_{1}=1.679$

Cu1-N1 $0.549 \quad \mathrm{~d}=1.973(8)$

$\mathrm{r}_{1}=1.751$

\section{$\mathrm{Cu} 22.098$}

$\mathrm{Cu} 2-\mathrm{O} 28 \quad 0.418 \quad \mathrm{~d}=2.002(5)$

$\mathrm{Cu} 2-\mathrm{O} 3 \quad 0.435 \quad \mathrm{~d}=1.987(5)$

Cu2-O6 $\quad 0.120 \quad d=2.464(5)$

$\mathrm{Cu} 2^{1}-\mathrm{O} 13 \quad 0.115 \mathrm{~d}=2.480(5)$

$\mathrm{Cu} 2-\mathrm{O} 26^{1} \quad 0.474 \quad \mathrm{~d}=1.955(5)$

$\mathrm{r}_{1}=1.679$

Cu2-N3 $0.537 \quad d=1.981(4)$

$\mathrm{r}_{1}=1.751$

\section{Cu3 2.112}

\begin{tabular}{|c|c|c|c|c|c|}
\hline $\mathrm{Cu} 3-\mathrm{O} 28$ & 0.401 & $\mathrm{~d}=2.017(6)$ & \multicolumn{3}{|c|}{$\mathrm{Cu} 41.036$} \\
\hline $\mathrm{Cu} 3-\mathrm{O} 2$ & 0.455 & $\mathrm{~d}=1.970(6)$ & $\mathrm{Cu} 4-\mathrm{O} 1$ & 0.053 & $\mathrm{~d}=2.592(5)$ \\
\hline $\mathrm{Cu} 3-\mathrm{O} 16^{1}$ & 0.479 & $\mathrm{~d}=1.951(5)$ & $\mathrm{Cu} 4-\mathrm{O} 1^{1}$ & 0.078 & $\mathrm{~d}=2.448(6)$ \\
\hline $\mathrm{Cu} 3-\mathrm{O} 22^{1}$ & 0.142 & $\mathrm{~d}=2.402(5)$ & \multicolumn{3}{|l|}{$\mathrm{r}_{1}=1.054$} \\
\hline $\mathrm{Cu} 3^{1}-\mathrm{O} 13$ & 0.116 & $\mathrm{~d}=2.475(6)$ & $\mathrm{Cu} 3-\mathrm{N} 4$ & 0.447 & $\mathrm{~d}=1.928(9)$ \\
\hline$r_{1}=1.679$ & & & $\mathrm{Cu} 3-\mathrm{N} 5$ & 0.458 & $\mathrm{~d}=1.919(9)$ \\
\hline $\mathrm{Cu} 3-\mathrm{N} 2$ & \multicolumn{2}{|l|}{0.523} & \multicolumn{3}{|l|}{$\mathrm{r}_{1}=1.63$} \\
\hline$r_{1}=1.751$ & & & & & \\
\hline
\end{tabular}

Cu5 1.26

Cu5-O5 $0.094 \quad d=2.378(6)$

$\mathrm{Cu}^{-}-\mathrm{O}^{2} \quad 0.101 \mathrm{~d}=2.353(6)$

$\mathrm{r}_{1}=1.054$

Cu5-N6 $0.474 \quad d=1.906(7)$ 
Cu5-N7 $\quad 0.600 \mathrm{~d}=1.819(8)$

$\mathrm{r}_{1}=1.63$

${ }^{[b]} \mathrm{Vi}=\Sigma \mathrm{Sij}=\Sigma \exp [(\mathrm{r} 1-\mathrm{rij}) / \mathrm{B}], \mathrm{r} 1$ is the bond length between atoms $\mathrm{i}$ and $\mathrm{j}$ (here $\mathrm{r} 1=1.679$ for $\mathrm{Cu}^{\mathrm{ii}}-\mathrm{O}$; $\mathrm{r} 1=1.751$ for $\mathrm{Cu}^{\mathrm{ii}-\mathrm{N}} ; \mathrm{r} 1=1.504$ for $\mathrm{Cu}^{\mathrm{i}}-\mathrm{O} ; \mathrm{r} 1=1.63$ for $\left.\mathrm{Cu}^{\mathrm{i}}-\mathrm{N}\right)$; $\mathrm{B}$ is a constant, the "universal parameter" $\sim 0.37 \AA$; Sij is the valence of a bond between atoms $\mathrm{i}$ and $\mathrm{j} ; \mathrm{Vi}$ is the sum of all bond valences of the bonds formed by a given atom $\mathrm{i}^{3}$

Table S4 Bond valence sum (BVS) analysis of metal ions in $\mathbf{3}^{[\mathrm{cc}]}$

Cu1 2.024

Cu1-O2 $0.444 \quad d=1.976(6)$

$\mathrm{Cu} 1-\mathrm{O} 3 \quad 0.387 \quad \mathrm{~d}=2.030(3)$

Cu1-O4 $0.129 \quad \mathrm{~d}=2.437(6)$

$\mathrm{Cu}^{2}-\mathrm{O} 4 \quad 0.129 \quad \mathrm{~d}=2.437(6)$

Cu1-O5 $0.447 \quad \mathrm{~d}=1.977(5)$

$\mathrm{r}_{1}=1.679$

Cu1-N1 $0.487 \quad \mathrm{~d}=2.017(7)$

$\mathrm{r}_{1}=1.751$
Cu2 2.049

$$
\begin{array}{lll}
\mathrm{Cu} 2-\mathrm{O} 21 & 0.397 & \mathrm{~d}=2.021(3) \\
\mathrm{Cu} 2-\mathrm{O} 9 & 0.428 & \mathrm{~d}=1.993(5) \\
\mathrm{Cu} 2-\mathrm{O} 14 & 0.118 & \mathrm{~d}=2.471(6) \\
\mathrm{Cu} 2{ }^{1}-\mathrm{O} 14 & 0.118 & \mathrm{~d}=2.471(6) \\
\mathrm{Cu} 2-\mathrm{O} 15 & 0.452 & \mathrm{~d}=1.973(6) \\
\mathrm{r}_{1}=1.679 & & \\
\mathrm{Cu} 2-\mathrm{N} 2 & 0.537 & \mathrm{~d}=1.981(6) \\
\mathrm{r}_{1}=1.751 & &
\end{array}
$$

\section{Cu3 1.060}

$$
\begin{array}{lll}
\text { Cu3-O18 } & 0.051 & d=2.604(17) \\
\text { Cu3-O6 } & 0.046 & d=2.664(16) \\
r_{1}= & 1.504 & \\
\text { Cu3-N5 } & 0.479 & d=1.902(8) \\
\text { Cu3-N7 } & 0.483 & d=1.899(8) \\
r_{1}= & 1.63
\end{array}
$$

${ }^{[\mathrm{c}]} \mathrm{Vi}=\Sigma \mathrm{Sij}=\Sigma \exp [(\mathrm{r} 1-\mathrm{rij}) / \mathrm{B}], \mathrm{r} 1$ is the bond length between atoms $\mathrm{i}$ and $\mathrm{j}$ (here $\mathrm{r} 1=1.679$ for $\mathrm{Cu}{ }^{\mathrm{ii}}-\mathrm{O} ; \mathrm{r} 1=1.751$ for $\mathrm{Cu}^{\mathrm{ii}}-\mathrm{N} ; \mathrm{r} 1=1.504$ for $\mathrm{Cu}^{\mathrm{i}}-\mathrm{O} ; \mathrm{rl}=1.63$ for $\left.\mathrm{Cu}^{\mathrm{i}}-\mathrm{N}\right)$; $\mathrm{B}$ is a constant, the "universal parameter" $\sim 0.37 \AA$; Sij is the valence of a bond between atoms $\mathrm{i}$ and $\mathrm{j}$; $\mathrm{Vi}$ is the sum of all bond valences of the bonds formed by a given atom $\mathrm{i}^{3}$

\section{References}

[1] O.V. Dolomanov, L. J. Bourhis, R. J. Gildea, J. A. K. Howard, H. Puschmann, J. Appl. Cryst. 2009, 42, 339-341.

[2] P. Vandersluis, A. L. Spek, Acta Crystallogr. A, 1990, 46, 194-201. 
[3] N. E. Brese, M. Okeeffe, Acta Crystallogr. B ,1991, 47, 192-197. 